\title{
Chondrosarcoma of the thyroid cartilage: a rare laryngeal malignancy
}

\author{
Lame $\mathrm{CA}^{1 *}$, Loum B ${ }^{1}$, Diallo TB ${ }^{1}$, Sarr ES ${ }^{2}$, Ndiaye $\mathrm{CB}^{1}$, Sissoko $\mathrm{B}^{1}$ and Faye MB ${ }^{1}$ \\ ${ }^{1}$ Department of ENT, Hopital Principal de Dakar, 1 Avenue Nelson Mandela, Dakar, Senegal \\ ${ }^{2}$ Department of Pathology, Hopital Principal de Dakar, 1 Avenue Nelson Mandela, Dakar, Senegal
}

\begin{abstract}
Laryngeal chondrosarcoma is a rare malignant tumor that arises from the cartilaginous structures of the larynx. It is a slow-growing neoplasm with high tendency to locoregional recurrence after treatment. Surgery is the primary treatment. Prognosis depends on histological differentiation degree and quality of surgical excision which can be very mutilating. Distant metastatis can occur in cases of histological high-grade tumors. From a new observation and a review of the literature, we discuss the clinical characteristics, therapeutic modalities and patient outcomes.
\end{abstract}

\section{Introduction}

Laryngeal chondrosarcomas are rare malignancies involving laryngeal cartilages. The cricoid cartilage represents the most commomly primary site. Treatment is usually based on surgical excision, with a favorable prognosis. We report the case of a patient who had a thyroid localization of a laryngeal chondrosarcoma with secondary pulmonary metastasis leading to death.

\section{Case report}

A 65-year-old man, print worker, diabetic, hypertensive with no alcoholism and no smoking history presented to our department with chronic dysphonia.

Clinical examination revealed a hard neck swelling at the right thyroid level. Nasofibroscopy showed an endolaryngeal submucosal mass, with fixation of the right vocal cord. CT scan (Figure 1) revealed a right supraglottic lesion which had spread into the pre-laryngeal space containing calcifications, thyroid cartilage destruction with right upper edge infiltration of the cricoid cartilage and right thyroid gland infiltration.

Endoscopic assessment found a right supraglottic submucosal bulging involving the right vestibular and false vocal cord. The overlay mucosa was regular. Deep biopsies were performed after incision of the laryngeal mucosa. Histological findings match the diagnosis of grade III laryngeal chondrosarcoma (Figure 2). Locoregional and general extension assessment was negative. Total laryngectomy with bilateral functional neck dissection and right lobo-isthmectomy were performed, followed by radiotherapy. After one year follow-up, the patient died from lung metastases.

\section{Discussion}

Laryngeal chondrosarcoma is an extremely rare cartilaginous malignant tumor. It accounts for 0.07 to $2 \%$ of laryngeal cancers and less than $1 \%$ of all sarcomas [1-4]. The primary site was most commonly the cricoid in $75 \%$ of cases, followed by the thyroid cartilage in $20 \%$, and epiglottis or arytenoids in 5\% [1]. Laryngeal chondrosarcomas appear around the age of 60 to 70 years with a clear predominance in males [1-5], as in our patient.

The etiopathogenesis of laryngeal chondrosarcoma has not yet been elucidated. The most likely mechanism would be a primary disorder of cartilage ossification. Some authors suggest chronic inflammation or ischemic changes in a preexisting chondroma $[1,4]$. Repeated laryngeal trauma, as well as anterior neck irradiation and teflon injection are also implicated [3,4].

As any laryngeal tumor, patients with laryngeal chondrosarcoma present various symptoms : dysphonia, dyspnea, dysphagia, stridor. Palpation may return to a hard anterior cervical mass when localized to the thyroid cartilage $[1,2]$. Our patient presented chronic dysphonia with a hard slowly-growing and painless neck swelling. The very slow evolution of this tumor explains average consultation time of 28 months found in the literature $[1,4,6,7]$.

During radiologic assessment, the performance of computed tomography (CT) usually shows a tumor process with different sizes calcifications inside, moderately enhanced after contrast injection, cartilage destruction and structure distortion [1,2]. CT also helps to precise the site and extent of tumor invasion which condition therapy. In our patient, CT showed a pre-laryngeal tumor containing calcifications, with thyroid cartilage destruction, upper edge cricoid infiltration and right thyroid gland infiltration.

MRI studies show T1 low-signal and T2 high-signal with characteristic mosaic pattern without providing much additionnal information $[2,3]$.

Correspondence to: Cheikh Ahmedou Lame, Department of ENT, Hopital Principal de Dakar, 1 Avenue Nelson Mandela, Dakar, Senegal, Tel: +221 33839 50 50; Email: cheikhlame@gmail.com

Key words: laryngeal chondrosarcoma, laryngeal cancer, laryngeal tumor, thyroid cartilage

Received: December 06, 2017; Accepted: December 23, 2017; Published: December 26, 2017 

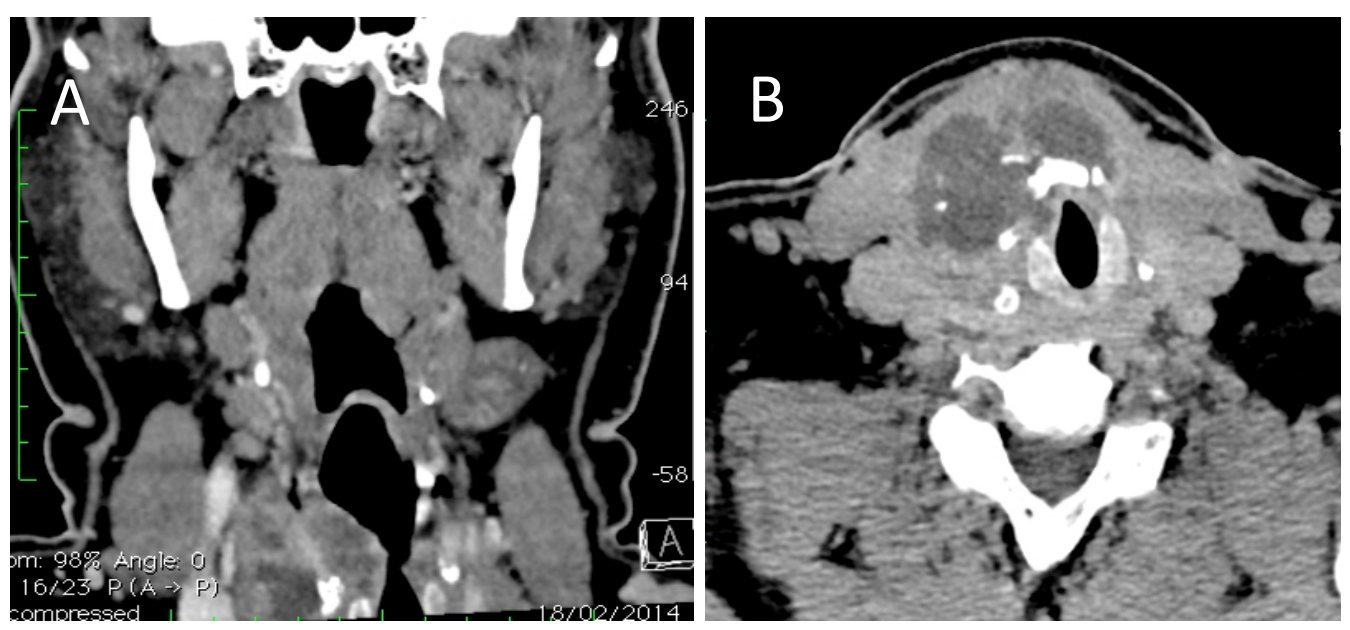

Figure 1. Coronal (A) and Axial (B) neck CT images showing a large tumor involving the prelaryngeal space with calcifications inside, right thyroid cartilage destruction and ipsilateral thyroid gland infiltration.
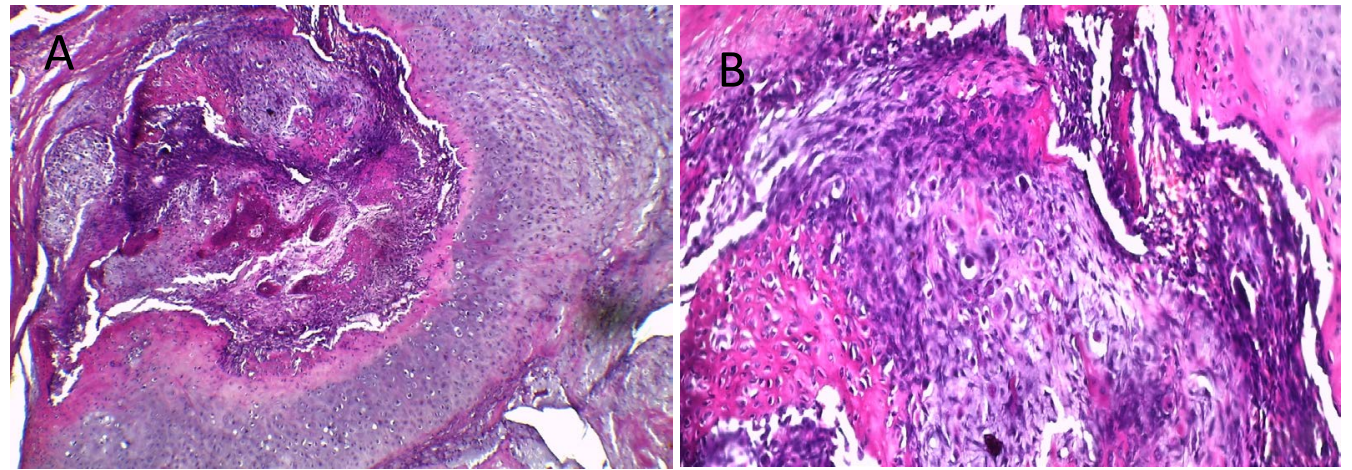

Figure 2. Pathology of the specimen revealing high-grade chondrosarcoma with proeminent cellularity and pleomorphism. (A) haematoxylin eosin staining HES $4 \times$, (B) haematoxylin eosin staining HES $10 \times$

PET scan is useful for histological grading, metastases detection and local relapse detection [3].

Endoscopy allows deep biopsies as chondrosarcomas are submucosal lesion [2,3,4,7]. Histological analysis discloses hyaline cartilage with giant cartilage cells, nuclear atypia, invasion/destruction of neighborhood structures. Three grade increasing malignancies are described according to Jaffe and Lichtenstein classification (1943) modified by Evans et al. [1,3]:

- Low grade (or grade I) chondrosarcomas with no mitotic activity, presence of small nuclei, chondroid intercellular space and frequent calcifications. Grade I tumors represent $67.8 \%$ according to Chin et al. [4].

- Intermediate grade (or grade II) chondrosarcomas with larger nuclei, myxoid intercellular space and a low mitotic index. Grade II tumors represent $23.5 \%$ [4].

- High grade (or grade III) chondrosarcomas with higher mitotic index and nuclear atypia. Much rarer, grade III tumors represent 3.8\% [4].

The standard treatment of laryngeal chondrosarcoma is mainly based on surgical excision [5,6,8]. Partial laryngectomy, preserving laryngeal function, is preferable when tumor location and size allow oncologic control. Conservative surgery limits morbidity, but local recurrences and postoperative stenosis are furthermore possible [9].

Partial laryngectomy can be performed by endoscopic or open technique. Endoscopic approach is reserved for small lesions. Laser techniques allow better control of bleeding.
Total laryngectomy is indicated in cases of very advanced lesions, in case of infilration of surrounding tissues, in advanced degree differentiated cancers, or in cases of recurrence $[3,4,9]$. Lymphnode dissection is not usual, ganglion involvement rarely occurs [6].

In our case, localization at the thyroid cartilage associated with extra laryngeal extension and doubt on a cricoidal lesion led us to perform total laryngectomy with neck dissection.

The place of radiotherapy is controversial in chondrosarcoma management. Chondrosarcomas were classically considered as radioresistant tumors [4,5,9]. But recent studies [5] have shown radiotherapy effectiveness, particularly in tumors where negative margins resection cannot be obtained. Radiotherapy is reserved for high-grade lesions, lymph node involvement, positive margins resection or local recurrence [5].

Chemotherapy is used for lesions with a high risk of metastasis [5].

Prognosis is usually good. However, it depends on histological differentiation degree and surgical excision quality [2,4]. Grade I-II degrees are slow-growing tumors with very low tendency to local recurrence and metastasis (7-18\%). Distant metastasis risk increases to $71 \%$ in grade III lesions. When metastases occur, the lung is the first organ to be affected [10].

\section{Conclusion}

Laryngeal chondrosarcoma is an uncommon entity with unknown etiology. Thyroid localization is rare. Diagnosis is difficult. 
Treatment is exclusively based on surgical excision, requiring a large surgery, sometimes mutilating. Metastasis, correlated with the tumor differentiation degree, are not exceptional.

\section{References}

1. Moerman M, Kreps B, Forsyth R (2009) Laryngeal Chondrosarcoma: an Exceptional Localisation of a not Unfrequent Bone Tumor. Sarcoma 2009: 394908. [Crossref]

2. Nao EE, Bozec A, Vallicioni J, Poissonnet G, Riss JC, et al. (2011) Laryngeal chondrosarcoma: report of two cases. Eur Ann Otorhinolaryngol Head Neck Dis 128: 191-193. [Crossref]

3. Oliveira JF, Branquinho FA, Monteiro AR, Portugal ME, Guimarães AM2 (2014) Laryngeal chondrosarcoma--ten years of experience. Braz J Otorhinolaryngol 80: 354358. [Crossref]

4. Chin OY, Dubal PM, Sheikh AB, Unsal AA, Park RC, et al. (2017) Laryngeal chondrosarcoma: A systematic review of 592 cases. Laryngoscope 127: 430-439. [Crossref]
5. Obeso S, Llorente JL, Díaz-Molina JP, Sánchez-Fernández R, Rodrigo JP, et al. (2010) Surgical treatment of head and neck chondrosarcomas. Acta Otorrinolaringol Esp 61 : 262-271. [Crossref]

6. Hu R, Xu W, Liu H, Chen X (2014) Laryngeal chondrosarcoma of the arytenoid cartilage presenting as bilateral vocal fold immobility: a case report and literature review. J Voice 28: 13-17. [Crossref]

7. Miloundja J, Lescanne E, Garand G, Vinikoff-Sonier C, Beutter P, et al. (2005) Chondrosarcoma of the cricoid. Ann Otolaryngol Chir Cervicofac 122: 91-96. [Crossref]

8. Perrot C, Cortese S, Eluecque H, Mastronicola R, Sergeant C, et al. (2013) Laryngea chondrosarcoma: Repeated laser and radiofrequency ablation in the palliative setting. Eur Ann Otorhinolaryngol Head Neck Dis 130: 91-93.

9. Oudidi A, Hachimi H, El Alami MN (2005)[Laryngeal chondrosarcoma. Cancer Radiother 9: 343-346. [Crossref]

10. Dominguez-Duran E, Menoyo-Bueno A, Gonzalez-Canton J, Abrante-Jiménez A (2014) Cutaneous Metastases of Laryngeal Chondrosarcoma. Acta Otorrinolaringol Esp 65: 373-374. [Crossref]

Copyright: $@ 2017$ Lame CA. This is an open-access article distributed under the terms of the Creative Commons Attribution License, which permits unrestricted use, distribution, and reproduction in any medium, provided the original author and source are credited. 